\title{
Role of mean platelet volume-to-lymphocyte ratio in the diagnosis of childhood appendicitis
}

\author{
Gulcin Bozlu, M.D., Associate Professor ${ }^{a}$, Asuman Akar, M.D. ${ }^{b}$, Fatma Durak, M.D. ${ }^{a}$ \\ and Necdet Kuyucu, M.D., Professor. ${ }^{b}$
}

\begin{abstract}
Introduction. Me a n platelet volume-to -lymphocyte ratio (MPVLR) has emerged as a novel parameter of inflammation and thrombosis. The aim was to evaluate whether MPVLR has a role in the diagnosis of childhood appendicitis.

Population and methods. Retrospectively reviewed the medical records up to 18 year with suspected acute appendicitis (AA) who underwent appendectomy between January 2016 and December 2017. Based on histopathological evaluation, the patients were classified into normal appendix, and simple and perforated appendicitis. Preoperative mean platelet volume (MPV), platelet distribution width (PDW), MPVLR, platelet-to-lymphocyte ratio (PLR), counts of platelets and white blood cells (WBC), and C-reactive protein (CRP) levels were measured.
\end{abstract}

Results. A total of 219 children with clinically suspected AA whounderwent surgical treatment for appendicitis were reviewed, and 100 genderage matched healthy subjects were included. Compared with the controls, the mean levels of MPVLR, PLR, WBC and CRP were significantly higher in the children underwent appendectomy $(p<0.001)$. These values were significantly higher in simpleappendicitis than in normal appendices $(p<0.01)$. Compared with simple appendicitis, these levels were significantly higher in the children with perforated appendicitis $(\mathrm{p}<0.001)$. The area under curves for MPVLR and PLR were 0.771 and 0.726 in the prediction of appendicitis and 0.693 and 0.722 in the prediction of perforation, respectively.

Conclusions. Increased level of MPVLR may be valuable for aiding the diagnosis of pediatric AA. In addition, it c a n helpdiscriminate simple and perforated appendicitis in children. Key words: acute appendicitis, child, diagnosis, mean platelet volume to/lymphocyte ratio.

http: / / dx.doi.org/10.5546/ aap.2019.eng.375

To cite: Bozlu G, Akar A, Durak F, Kuyucu N. Role of mean platelet volume-to-lymphocyte ratio in the diagnosis of childhood appendicitis. Arch Argent Pediatr 2019;117(6):375-380.

\section{INTRODUCTION}

Acute appendicitis (AA) is the most common reason for urgent abdominal operation and approximately $20-30 \%$ of children with acute abdominal pain admitted to pediatric emergency department have AA. ${ }^{1}$ A delay in diagnosis of AA may result in life-threatening complications such as perforation and peritonitis. In children, this condition often presents late or with atypical features. Therefore, careful diagnosis and prompt management of pediatric appendicitis is imperative. ${ }^{2}$ Although several laboratory tests and imaging investigations have been used for fast and accurate diagnosis, they had limitations to differentiate appendicitis from other causes of abdominal pain. ${ }^{3}$

Recently, there has been growing interest in simple hematological parameters, such as mean platelet volume (MPV) and platelet-tolymphocyte ratio (PLR), which are thought to be useful markers of the severity of the systemic inflammatory response. Mean platelet volumeto-lymphocyte ratio (MPVLR) is a parameter calculated and provided by automatic blood count equipment during routine blood counts. Hudzik et al. ${ }^{4}$ demonstrated that MPVLR has emerged as a novel and readily available marker of inflammation and trombosis in adults. In addition, recent studies have been reported that MPVLR level has clinical implications in various pathologies such as systemic inflammation, myocardial infarction and impaired coronary collateral circulation..$^{5,6}$ On the other hand, no study has evaluated the role of MPVLR in children and the diagnostic value of MPVLR in children with AA. 
In the present study, we aimed to evaluate the role of MPVLR in the diagnosis of childhood appendicitis.

\section{POPULATION AND METHODS}

This study was conducted after obtaining an approval from Mersin University Clinical Research Ethics Committee (the protocol number that was attributed by the ethics committee was: $32 / 2018$ and the date of approval was January 18, 2018). We retrospectively reviewed the medical records of the children aged 18 year or younger with clinically suspected AA who underwent appendectomy at our institution between January 2016 and December 2017. Gender-age matched healthy subjects, who had been admitted to pediatric clinic and did not have any disease, were taken as a control group (we have a healthy outpatients clinic in our Pediatrics Department. Periodically, healthy children are given control tests such as hemogram and urine tests). Children with incomplete medical records, with known hematological diseases (platelet or coagulation abnormalities), allergic diseases, malignant and inflammatory diseases and receiving drugs that can impact the hematological parameters were excluded from the study.

Patient characteristics and preoperative laboratory parameters including MPV, platelet distribution width (PDW), and counts of platelets and white blood cells (WBC), and C-reactive protein (CRP) levels were measured. The MPVLR and PLR were calculated by dividing MPV and platelet count by the lymphocyte count respectively. Based on postoperative histopathological evaluation, the patients were classified into normal appendix, simple appendicitis and perforated appendicitis. The levels of preoperative laboratory parameters were compared.
Statistical analyses: The sample size was calculated with MedCalc 18.9 software (number of cases in patient group was 187 children, and number of cases in control group was 94 children for $95 \%$ CI). All continuous variables are expressed as mean \pm standard deviation. Data of patients and healthy controls were compared. Preoperative laboratory parameters of the patients who underwent appendectomy were also compared with regard to histopathological evaluation. The $t$ test, analysis of variance and receiver operating characteristic (ROC) curve were used for statistical analysis. Multiple comparisons were made using one-way ANOVA with post-hoc Tukey's test. The ROC curves were used to examine the diagnostic value of MPVLR and PLR in suspected AA group. The area under curves (AUC) were calculated for these parameters. The appropriate cutoff values of MPVLR and PLR were determined using maximum sum of sensitivity and specificity. Sensitivity, specificity, positive predictive value (PPV), negative predictive value (NPV) and overall accuracy rates were calculated. Multivariate logistic regression models were used to identify predictors of appendix perforation in children with AA. The results were considered statistical significance if $p$ values were less than 0.05 .

\section{RESULTS}

After exclusion, a total of 219 children who undrewent appendectomy and 100 sex-age matched healthy subjects, who had been admitted to pediatric clinic and did not have any disease, were included. The characteristics of the patients and controls are presented in Table 1. There was no significant difference in age, gender, platelet counts, MPV and PDW between the patients and controls ( $p>0.05)$. Compared with

TABLE 1. Comparison of characteristic of patients and controls

\begin{tabular}{lccl}
\hline & Patient $(\mathbf{n}=\mathbf{2 1 9})$ & Control (n= 100) & $p$ \\
\hline Age (year) & $10.72 \pm 4.31$ & $10.70 \pm 4.12$ & 0.768 \\
Gender $($ male $/$ female $)$ & $137 / 82$ & $62 / 38$ & 0.356 \\
Platelet $\left(x 10^{3} / \mu \mathrm{L}\right)$ & $295.51 \pm 89.78$ & $298.47 \pm 109.49$ & 0.245 \\
MPV $(\mathrm{fL})$ & $9.85 \pm 1.05$ & $9.78 \pm 0.95$ & 0.432 \\
PDW $(\%)$ & $12.75 \pm 2.65$ & $12.56 \pm 2.88$ & 0.546 \\
MPVLR & $6.77 \pm 3.67$ & $3.69 \pm 2.33$ & $<0.001$ \\
PLR & $197.23 \pm 94.53$ & $136.77 \pm 75.51$ & $<0.001$ \\
WBC $\left(x 10^{3} / \mu \mathrm{L}\right)$ & $14.56 \pm 5.23$ & $9.25 \pm 3.12$ & $<0.001$ \\
CRP $(\mathrm{mg} / \mathrm{dL})$ & $48.95 \pm 70.31$ & $2.16 \pm 2.67$ & $<0.001$ \\
\hline
\end{tabular}

MPV, mean platelet volume; PDW, platelet distribution width; MPVLR, mean platelet volume-to-lymphocyte ratio; PLR, platelet-to-lymphocyte ratio; WBC, white blood cell; CRP, C-reactive protein. 
healthy controls, a significant increase in MPVLR, PLR, WBC and CRP was noted in children who underwent appendectomy $(\mathrm{p}<0.001$, Table 1$)$.

The pathological diagnoses were normal appendices in $46(21 \%)$, simple appendicitis in $141(64.3 \%)$ and perforated in $32(14.6 \%)$ children. The parameters for patients with normal appendices, simple appendicitis and perforated appendicitis were displayed in Table 2. MPVLR, PLR, WBC and CRP levels were significantly higher in simple appendicitis than in the children with normal appendices $(p<0.01)$. Compared with simple appendicitis, MPVLR, PLR, WBC and CRP levels were significantly higher in the children with perforated appendicitis (Table 2, $\mathrm{p}<0.001$ ).
The AUCs for MPVLR and PLR were 0.771 and 0.726 in the prediction of appendicitis (Figure 1) and 0.693 and 0.722 in the prediction of perforation (Figure 2), respectively. The sensitivity, specificity, PPV, NPV and accuracy values were detailed in Table 3 . In the multivariate analysis discriminating perforated from nonperforated appendicitis, MPVLR was significant parameter for predicting appendiceal perforation (Table 4).

\section{DİSCUSSION}

To our knowledge, diagnostic value of MPVLR in children with AA has not been studied before. This study showed that children who underwent appendectomy had significantly higher levels of

TABLE 2. Comparison of blood parameters among patients with normal appendices, simple appendicitis and perforated appendicitis.

\begin{tabular}{|c|c|c|c|c|c|}
\hline & $\begin{array}{l}\text { Normal appendices } \\
\quad(n=46)\end{array}$ & $\begin{array}{c}\text { Simple appendicitis } \\
(\mathrm{n}=141)\end{array}$ & $\begin{array}{l}\text { Perforated appendicitis } \\
\qquad(\mathrm{n}=32)\end{array}$ & ${ }^{\mathrm{a}} p$ & ${ }^{\mathrm{b}} p$ \\
\hline Platelet $\left(\times 10^{3} / \mu \mathrm{L}\right)$ & $300.96 \pm 74.85$ & $299.33 . \pm 92.95$ & $298.75 \pm 95.97$ & 0.224 & 0.535 \\
\hline MPV(fL) & $9.82 \pm 0.95$ & $9.78 \pm 0.88$ & $9.84 \pm 1.09$ & 0.663 & 0.689 \\
\hline PDW $(\%)$ & $12.58 \pm 2.54$ & $12.88 \pm 2.71$ & $12.36 \pm 2.45$ & 0.505 & 0.389 \\
\hline MPVLR & $4.73 \pm 1.91$ & $6.29 \pm 3.01$ & $9.43 \pm 5.70$ & 0.001 & 0.001 \\
\hline PLR & $146.70 \pm 60.41$ & $186.41 \pm 91.18$ & $250.90 \pm 92.26$ & 0.001 & 0.001 \\
\hline $\mathrm{WBC}\left(\times 10^{3} / \mu \mathrm{L}\right)$ & $11.74 \pm 3.87$ & $14.18 \pm 5.10$ & $16.53 \pm 5.72$ & 0.001 & 0.019 \\
\hline $\mathrm{CRP}(\mathrm{mg} / \mathrm{dL})$ & $19.65 \pm 29.17$ & $40.20 \pm 63.50$ & $101.02 \pm 86.36$ & 0.012 & 0.001 \\
\hline
\end{tabular}

${ }^{a} \mathrm{p}$ : Normal appendices vs. simple appendicitis.

${ }^{\mathrm{b}} \mathrm{p}$ : Simple appendicitis vs. perforated appendicitis.

MPV, mean platelet volume; PDW, platelet distribution width; MPVLR, mean platelet volume-to-lymphocyte ratio;

PLR, platelet-to-lymphocyte ratio; WBC, white blood cell; CRP, C-reactive protein.

FIGURE 1. Receiver operating characteristic curves for MPVLR and PLR in predicting acute appendicitis

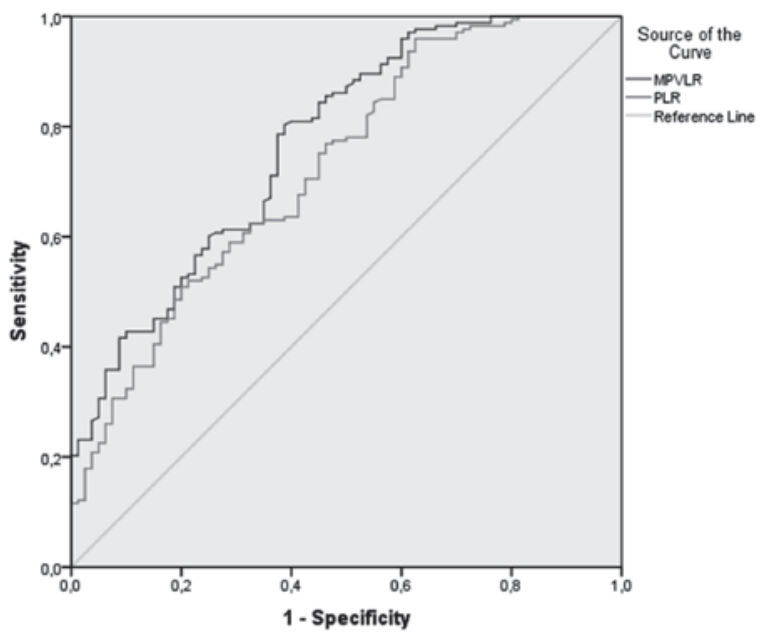

MPVLR, mean platelet volume-to-lymphocyte ratio; PLR, platelet-to-lymphocyte ratio.
FIgURE 2. Receiver operating characteristic curves for $M P V L R$ and PLR in predicting perforated appendicitis

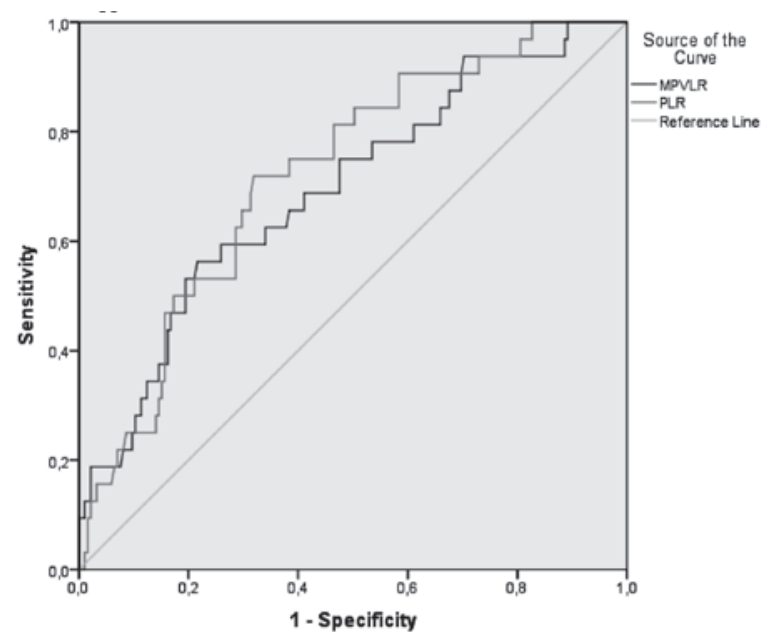

MPVLR, mean platelet volume-to-lymphocyte ratio; PLR, platelet-to-lymphocyte ratio. 
MPVLR, PLR, WBC and CRP than the control subjects. The results of our study also indicated that the levels of MPVLR, PLR, WBC and CRP in children with simple and perforated appendicitis were significantly increased than did those with normal appendix. Furthermore, it was shown that those parameters were significantly higher in children with perforated appendicitis compared to the children without.

Although its classical signs and symptoms are well known, the clinical diagnosis of AA is still problematic in children. ${ }^{1,2} \mathrm{~A}$ delay in diagnosis is associated with perforation and increased complication rate. On the other hand, misdiagnosis appendicitis may lead to unnecessary surgery. ${ }^{7}$ In clinical practice, history and clinical examination remain the cornerstone of diagnosis, but, several studies have been focused on the diagnostic value of laboratory markers in acute appendicitis. ${ }^{3,8,9}$

MPV is a marker of platelet function and activation, can be easily measured in routine complete blood count test cycle. It has been demonstrated that MPV is a laboratory marker of inflammatory conditions. ${ }^{10}$ Firstly, Labara et al., ${ }^{11}$ found that a statistically significant decrease in MPV was noted in adult patients with AA compared with healthy controls. They suggested that MPV may guide the management of patients suspected to have AA. The mechanism for decreased MPV in inflammatory diseases remains unclear. It may be the presence of a defect in the regulation of thrombopoiesis in inflammatory conditions. ${ }^{10,12}$ On the other hand, limited number of studies reported the role of MPV in diagnosis of AA in children. They have reported contradictory results. Bilici et al., ${ }^{13}$ indicated that MPV was significantly lower in pediatric AA patients compared to the control. Contrarily, Uyanık et al., ${ }^{14}$ detected that MPV has no diagnostic value in pediatric AA cases. However, we found that there was no significant difference between AA cases and control subjects with respect to MPV levels. It has been suggested that clinical utility of MPV to discriminate AA from other acute abdomen have to be analyzed separately among genders. ${ }^{15}$ In our study, there was no significant difference in gender between the patients and controls.

TABLE 3. Sensitivity, specificity, PPV and NPV rates for MPVLR and PLR for predicting acute appendicitis and perforated appendicitis

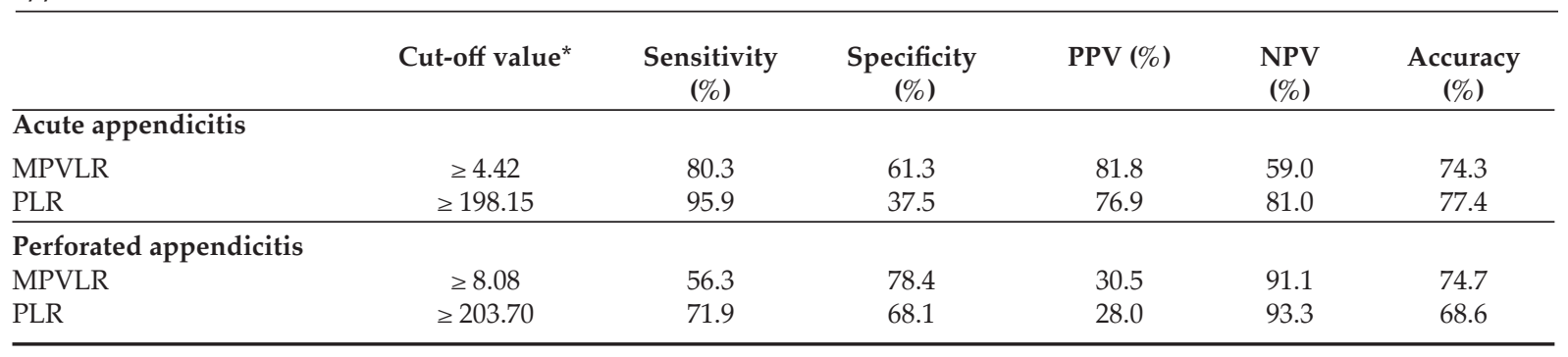

${ }^{*}$ Cut-off values have been calculated for the biggest Youden index (Sensitivity + Specificity)-1 in ROC analysis.

MPVLR, mean platelet volume-to-lymphocyte ratio; PLR, platelet-to-lymphocyte ratio; PPV, positive predictive value;

NPV, negative predictive value.

TABLE 4. Multivariate analysis with parameters for predicting appendix perforation

\begin{tabular}{lcccc}
\hline & \multirow{2}{*}{ Statistical significance } & Exp (B) & \multicolumn{2}{c}{$95 \%$ C.I. for EXP (B) } \\
& & & Upwer & 1.001 \\
\hline Age & 0.068 & 0.984 & 0.967 & 7.583 \\
Gender & 0.296 & 2.022 & 0.539 & 1.046 \\
Platelet & 0.877 & 1.003 & 0.962 & 20.555 \\
MPV & 0.669 & 1.718 & 0.144 & 2.115 \\
PDW & 0.530 & 0.702 & 0.233 & 4.023 \\
MPVLR & 0.032 & 1.070 & 0.962 & 1.052 \\
PLR & 0.793 & 1.006 & 0.288 & 6.128 \\
WBC & 0.716 & 1.009 & 1.000 & 1.019 \\
CRP & 0.056 & & \\
\hline
\end{tabular}

Abreviations: MPV, mean platelet volume; PDW, platelet distribution width; MPVLR, mean platelet volume-to-lymphocyte ratio; PLR, platelet-to-lymphocyte ratio; WBC, white blood cell; CRP, C-reactive protein. 
Blood MPVLR levels were calculated by dividing the MPV value by the lymphocyte count. MPVLR has emerged as a novel laboratory test of inflammation and trombosis. There has been relatively little research on the usefulness of MPVLR. Hudzik et al., ${ }^{4}$ investigated the prognostic significance of MPVLR in diabetic patients with in ST elevation myocardial infarction. They concluded that MPVLR plays a pivotal role in developing intravascular thrombus in ST elevation myocardial infarction and elevated MPVLR is an independent risk factor of mortality in patients with acute myocardial infarction. Increased levels of MPVLR are associated with angiographic no-reflow and impaired coronary collateral circulation. ${ }^{5,6}$ More recently, it has been reported that MPVLR may be helpful in predicting coronary artery lesions in patients with Kawasaki disease. ${ }^{16}$

The role of MPVLR has not been defined in pediatric AA and the findings from the studies mentioned above led us to evaluate whether MPVLR may have a value in children with AA. We found a significantly higher MPVLR level in children with AA compared to control. Our results also showed that the mean MPVLR level of perforated AA was significantly higher than children with non-perforated AA. It has been reported that ROC analysis revealed moderate diagnostic value of MPVLR in prediction of hospital death in diabetic patients with in ST elevation myocardial infarction in patients. ${ }^{4}$ In our study, ROC curve analysis revealed that AUCs for MPVLR were 0.771 and 0.693 in the prediction of acute appendicitis and perforation of appendicitis respectively.

Platelets are cells that help in modulating various inflammatory conditions; therefore, changes in PLR may be a prognostic indicator of acute infection, cardiovascular events and cancer. ${ }^{17,18}$ There are only a few studies examining the relationship between PLR and AA. Nazik et al., ${ }^{19}$ found a significantly higher PLR level in pediatric patients with AA compared with in healthy controls. In the pregnant women, the level of PLR was significantly higher in appendectomy group compared with healthy control group. ${ }^{20}$ In the current study, PLR value in children with AA was statistically significantly higher than in control subjects, which was in accordance with results reported in the previous studies. Moreover, we also found that pediatric perforated appendicitis group had a significantly higher PLR than simple appendicitis group. In this study, AUCs for PLR were 0.726 and 0.722 in the prediction of acute appendicitis and perforation of appendicitis respectively.

There were limitations to our study. It was a retrospective study and our findings may need to be confirmed in prospective studies. However, we were unable to analyze the correct diagnoses of the patients with normal appendix. Sample size analyses may be more appropriate to have children with confirmed appendicitis versus children with normal appendices or different severity of appendicitis. On the other hand, our study has several strengths as well. Ultrasound imaging is essential for the differential diagnosis of children with AA and all of the children had ultrasonographic evaluation. Despite the limitations, we present the first study to examine the value of MPVLR in pediatric AA. The greatest advantage with using MPVLR and PLR is their low cost of evaluation.

\section{CONCLUSIONS}

In conclusion, this is the first study that has been focused on the role of MPVLR in children with AA. We demonstrated that increased level of MPVLR may be valuable for aiding the diagnosis of pediatric AA. Moreover, MPVLR may serve to aid in discriminating between simple and perforated appendicitis in children.

\section{Acknowledgements:}

We would like to acknowledge Prof. Dr. Ali Nayci (Head of Pediatric Surgery Department) who helped in data collection.

\section{REFERENCES}

1. Rentea RM, Peter SD, Snyder CL. Pediatric appendicitis: state of the art review. Pediatr Surg Int. 2017;33(3):269-83.

2. Rothrock SG, Pagane J. Acute appendicitis in children: emergency department diagnosis and management. Ann Emerg Med. 2000; 36(1):39-51.

3. Benabbas R, Hanna M, Shah J, Sinert R. Diagnostic accuracy of history, physical examination, laboratory tests, and point-of-care ultrasound for pediatric acute appendicitis in the emergency department: a systematic review and meta-analysis. Acad Emerg Med. 2017; 24(5):523-51.

4. Hudzik B, Szkodziński J, Lekston A, Gierlotka M, et al. Mean platelet volume-to-lymphocyte ratio: a novel marker of poor short- and long-term prognosis in patients with diabetes mellitus and acute myocardial infarction. J Diabetes Complications. 2016; 30(6):1097-102.

5. KurtulA,AcikgozSK. Usefulness of mean platelet volumeto-lymphocyte ratio for predicting angiographic no-reflow and short-term prognosis after primary percutaneous coronary intervention in patients with st-segment elevation myocardial infarction. Am J Cardiol. 2017; 120(4):534-41.

6. Ornek E, Kurtul A. Relationship of mean platelet volume to lymphocyte ratio and coronary collateral circulation in patients with stable angina pectoris. Coron Artery Dis. 2017; 28(6):492-7. 
7. Dubrovsky G, Rouch J, Huynh N, Friedlander S, et al. Clinical and socioeconomic factors associated with negative pediatric appendicitis. J Surg Res. 2017; 218:322-8.

8. Shogilev DJ, Duus N, Odom SR, Shapiro NI. Diagnosing appendicitis: evidence-based review of the diagnostic approach in 2014. West J Emerg Med. 2014; 15(7):859-71.

9. Saucier A, Huang EY, Emeremni CA, Pershad J. Prospective evaluation of a clinical pathway for suspected appendicitis. Pediatrics. 2014; 133(1):e88-95.

10. Gasparyan AY, Ayvazyan L, Mikhailidis DP, Kitas GD. Mean platelet volume: a link between thrombosis and inflammation? Curr Pharm Des. 2011; 17(1):47-58.

11. Albayrak Y, Albayrak A, Albayrak F, Yildirim R, et al. Mean platelet volume: a new predictor in confirming acute appendicitis diagnosis. Clin Appl Thromb Hemost. 2011; 17(4):362-6.

12. Liu R, Gao F, Huo J, YiQ. Study on the relationship between mean platelet volume and platelet distribution width with coronary artery lesion in children with Kawasaki disease. Platelets. 2012; 23(1):11-6.

13. Bilici S, Sekmenli T, Goksu M, Melek M, Avci V. Mean platelet volume in diagnosis of acute appendicitis in children. Afr Health Sci. 2011; 11(3):427-32.

14. Uyanik B, Kavalci C, Arslan ED, Yilmaz F, et al. Role of mean platelet volume in diagnosis of childhood acute appendicitis. Emerg Med Int. 2012; 2012:823095.
15. Yang JJ, Cho SY, Ahn HJ, Lee HJ, et al. Mean platelet volume in acute appendicitis: a gender difference. Platelets. 2014; 25(3):226-7.

16. Bozlu G, Karpuz D, Hallioglu O, Unal S, Kuyucu N. Relationship between mean platelet volume-to-lymphocyte ratio and coronary artery abnormalities in Kawasaki disease. Cardiol Young. 2018; 28(6):832-6.

17. Kawamura Y, Takeshita S, Kanai T, Yoshida Y, Nonoyama S. The combined usefulness of the neutrophil-to-lymphocyte and platelet-to-lymphocyte ratios in predicting intravenous immunoglobulin resistance with Kawasaki disease. J Pediatr. 2016; 178:281-4.e1.

18. Yodying H, Matsuda A, Miyashita M, Matsumoto S, et al. Prognostic significance of neutrophil-to-lymphocyte ratio and platelet-to-lymphocyte ratio in oncologic outcomes of esophageal cancer: a systematic review and meta-analysis. Ann Surg Oncol. 2016; 23(2):646-54.

19. Nazik S, Avci V, Küskü Kiraz Z. Ischemia-modified albumin and other inflammatory markers in the diagnosis of appendicitis in children. Ulus Travma Acil Cerrahi Derg. 2017; 23(4):317-21.

20. Yazar FM, Bakacak M, Emre A, Urfalioglu A, et al. Predictive role of neutrophil-to-lymphocyte and platelet-to-lymphocyte ratios for diagnosis of acute appendicitis during pregnancy. Kaohsiung J Med Sci. 2017; 31(11):591-6. 